\title{
Efficacy of omega-3 polyunsaturated fatty acids on hormones, oxidative stress, and inflammatory parameters among polycystic ovary syndrome: a systematic review and meta-analysis
}

\author{
Jialing Yuan ${ }^{1,2}$, Xuemei Wen ${ }^{3}$, Ming Jia ${ }^{4}$ \\ ${ }^{1}$ Department of Obstetrics and Gynecology, West China Second University Hospital, Sichuan University, Chengdu, China; ${ }^{2}$ The Key Laboratory \\ of Birth Defects and Related Diseases of Women and Children (Sichuan University), Ministry of Education, Chengdu, China; ${ }^{3}$ Department of \\ Anesthesiology, the General Hospital of Western Theater Command, Chengdu, China; ${ }^{4}$ Shaanxi Institution of Traditional Chinese Medicine and \\ Pharmacology, Xi'an, China \\ Contributions: (I) Conception and design: All authors; (II) Administrative support: None; (III) Provision of study materials or patients: None; (IV) \\ Collection and assembly of data: All authors; (V) Data analysis and interpretation: All authors; (VI) Manuscript writing: All authors; (VII) Final \\ approval of manuscript: All authors. \\ Correspondence to: Ming Jia. Shaanxi Institution of Traditional Chinese Medicine and Pharmacology, Xi'an 710003, China. \\ Email: bianjibu3721@163.com.
}

Background There is a close relationship between hormones, oxidative stress, and inflammatory factors and polycystic ovary syndrome (PCOS). This meta-analysis was conducted to evaluate the changes in hormones, oxidative stress, and inflammatory factors of PCOS patients who were supplemented with omega-3 polyunsaturated fatty acids (n-3 PUFAs).

Methods: The databases of PubMed, Cochrane Library, Embase, and Web of Science were searched from inception to February 2021. We have included all randomized controlled trials (RCTs) that reported the n-3 PUFA treatment in PCOS. Weighted mean differences (WMD) and 95\% confidence interval (CI) were calculated, and either the fixed effects model or random effects model was used.

Results: 314 studies were initially identified, and 10 RCTs with 610 patients were finally included in the current study. Results suggested that PCOS patients with n-3 PUFAs supplementation may have a reduction in C-reactive protein (CRP; $-8.97 \mathrm{mg} / \mathrm{dL} ; 95 \% \mathrm{CI}:-17.66$ to $-0.28 \mathrm{mg} / \mathrm{dL} ; \mathrm{P}=0.04 ; \mathrm{I}^{2}=99 \%$ ); serum malondialdehyde (MDA; $-0.40 \mathrm{mg} / \mathrm{dL} ; 95 \% \mathrm{CI}:-0.56$ to $-0.25 \mathrm{mg} / \mathrm{dL} ; \mathrm{P}<0.00001 ; \mathrm{I}^{2}=42$ ); luteinizing hormone (LH; $-1.33 \mathrm{mg} / \mathrm{dL} ; 95 \% \mathrm{CI}:-2.63$ to $-0.04 \mathrm{mg} / \mathrm{dL} ; \mathrm{P}=0.04 ; \mathrm{I}^{2}=0 \%$ ); serum total testosterone (TT; $-0.11 \mathrm{mg} / \mathrm{dL}$; $95 \% \mathrm{CI}:-0.18$ to $-0.04 \mathrm{mg} / \mathrm{dL} ; \mathrm{P}=0.02 ; \mathrm{I}^{2}=73 \%$ ); and an increase in total antioxidant capacity (TAC; $72.24 \mathrm{mg} / \mathrm{dL} ; 95 \% \mathrm{CI}: 22.32$ to $122.16 \mathrm{mg} / \mathrm{dL} ; \mathrm{P}=0.005 ; \mathrm{I}^{2}=50 \%$ ) and serum sex hormone binding globulin (SHBG; $0.68 \mathrm{mg} / \mathrm{dL} ; 95 \%$ CI: 0.06 to $1.31 \mathrm{mg} / \mathrm{dL} ; \mathrm{P}=0.03 ; \mathrm{I}^{2}=0 \%$ ).However, no effect on glutathione (GSH; $-12.63 \mathrm{mg} / \mathrm{dL} ; 95 \% \mathrm{CI}:-50.34$ to $25.07 \mathrm{mg} / \mathrm{dL} ; \mathrm{P}=0.51 ; \mathrm{I}^{2}=56 \%$ ), dehydroepiandrosterone sulfate (DHEAS; $-0.01 \mathrm{mg} / \mathrm{dL} ; 95 \% \mathrm{CI}:-1.53$ to $1.50 \mathrm{mg} / \mathrm{dL} ; \mathrm{P}=0.99 ; \mathrm{I}^{2}=78 \%$ ), free androgen index (FAI; $0.00 \mathrm{mg} / \mathrm{dL}$; $95 \% \mathrm{CI}:-0.03$ to $0.03 \mathrm{mg} / \mathrm{dL}$; $\mathrm{P}=0.99 ; \mathrm{I}^{2}=0 \%$ ), or follicle-stimulating hormone (FSH; $0.37 \mathrm{mg} / \mathrm{dL}$; $95 \% \mathrm{CI}:-0.55$ to $1.29 \mathrm{mg} / \mathrm{dL} ; \mathrm{P}=0.43 ; \mathrm{I}^{2}=61$ ) was found.

Conclusions: This meta-analysis showed that supplementation of n-3 PUFAs in PCOS women can significantly improve CRP, MDA, LH, TT, TAC, and SHBG, but did not affect the concentrations of GSH, DHEAS, FAI, or FSH.

Keywords: Omega-3 polyunsaturated fatty acids (n-3 PUFAs); meta-analysis; polycystic ovary syndrome (PCOS); hormones; oxidative stress; inflammatory parameters

Submitted Jun 15, 2021. Accepted for publication Aug 17, 2021.

doi: 10.21037/apm-21-2018

View this article at: https://dx.doi.org/10.21037/apm-21-2018 


\section{Introduction}

Irregular menstruation and skin signs of androgen excess are commonly encountered in women of reproductive age. Polycystic ovary syndrome (PCOS) is the most common cause of these symptoms in adult women and can also be diagnosed in adolescents. It is the most common endocrine disorder, is accompanied by a multitude of conditions, and affects $6-20 \%$ of reproductive-age women $(1,2)$. Hyperandrogenism and its related consequences are notable clinical features of PCOS, and is present in up to $50-75 \%$ of women with PCOS (3). Hyperandrogenism also increases the risk of cardiovascular and cerebrovascular diseases, type 2 diabetes, endometrial cancer, and other diseases $(4,5)$. Meanwhile, studies have shown that hyperandrogenemia, inflammation, and oxidative damage play a key role in the pathophysiology of PCOS $(6,7)$.

The hormonal imbalance in PCOS patients can influence their reproductive, metabolic, and psychological health, contributing to irregular menstrual cycles, infertility and complications in pregnancy, insulin resistance (IR) in muscle and adipose tissue, hyperinsulinemia, anxiety, and depression $(8,9)$. The role of oxidative stress and inflammation in PCOS has been the subject of several studies. According to those studies, low-grade inflammation cooperates with IR and hyperandrogenism to constitute an interactive continuum acting on the pathophysiology of PCOS $(10,11)$.

Lifestyle and weight loss remain the first-line therapy of PCOS treatments; however, it is difficult for most patients to achieve and maintain the required level of weight loss in the long term. Nutritional intervention for women with polycystic ovary syndrome has attracted attention. In recent years, studies have shown that dietary fat is currently considered as a biological regulator affecting metabolism, and $\omega$ - Polyunsaturated fatty acids (n-3PUFA) may be the most effective metabolic fatty acid regulator (12-15). Animal studies shown that n-3 PUFAs can prevent IR by activating PPARa, reducing inflammation, and depositing fat in insulin-sensitive tissues (16). But, the effect of n-3 PUFA treatment on hormones, oxidative stress, and inflammation in PCOS patients are conflicting.

Considering the current controversy, we designed a randomized controlled trial (RCT)-based study to investigate the effects of supplementation with n-3 PUFAs on hormones, oxidative stress, and inflammation in women with PCOS.

We present the following article in accordance with the
PRISMA reporting checklist (available at https://dx.doi. org/10.21037/apm-21-2018).

\section{Methods}

\section{Protocol and registration}

The systematic review and meta-analyses were conducted in accordance with the PRISMA guidelines (17).

\section{Search strategy}

The databases of Web of science, the Cochrane Library, Embase, and MEDLINE were searched from their inception to February 2021 by 2 authors independently. The search keywords were the following: "omega-3 polyunsaturated fatty acids" OR "omega-3" OR "EPA" OR "DHA" OR "n-3 PUFA" AND ("PCOS" OR "polycystic ovary syndrome"). The investigators also conducted a manual search of the reference list of cited articles and their comments for further review.

\section{Selection criteria}

According to the selection criteria, 2 authors reviewed the articles independently.

Inclusion criteria: (I) population-patients of any age, or nationality diagnosed with PCOS; (II) interventionsomega-3 fatty acids of any dose and route of administration; (III) control group-placebo or other active agents; (IV) measurement of results-serum C-reactive protein (CRP), glutathione (GSH), malondialdehyde (MDA), total antioxidant capacity (TAC), dehydroepiandrosterone sulfate (DHEAS), free androgen index (FAI), follicle-stimulating hormone (FSH), luteinizing hormone (LH), sex hormone binding globulin (SHBG), and total testosterone (TT); (V) study type-RCT with no restriction regarding follow-up time or sample size.

Exclusion criteria: (I) not an RCT; (II) raw data not evaluated; (III) duplicate publications; (IV) letters to editors, conference papers, and articles provided only in the form of abstracts.

\section{Data extraction}

According to the inclusion and exclusion criteria, 2 reviewers independently reviewed all identified data and deleted duplicate articles. These reviewers screened titles, 


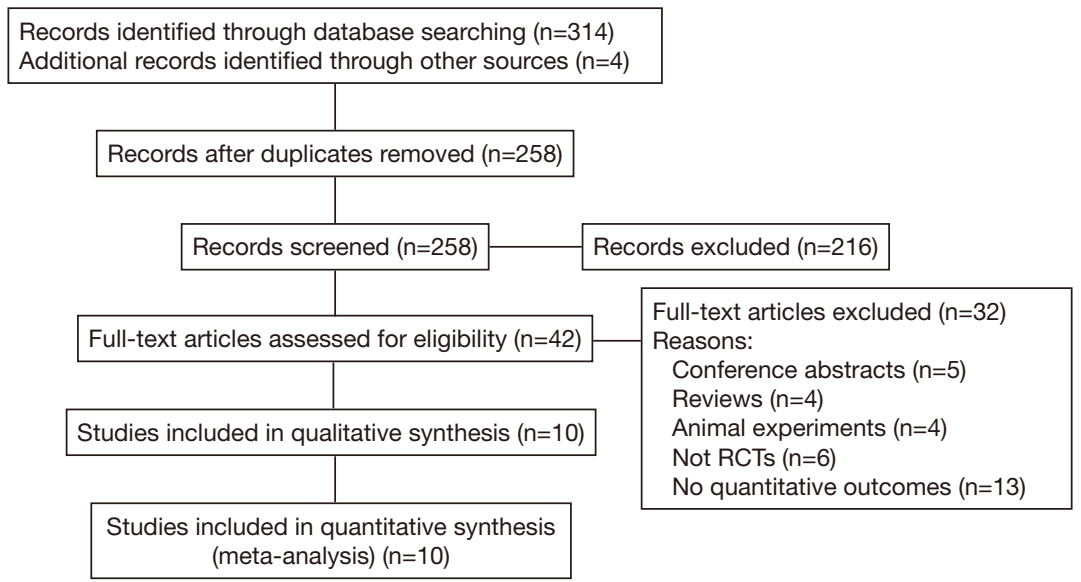

Figure 1 Study selection flow chart. RCT, randomized controlled trial.

abstracts, and full texts; extracted data independently; and resolved any differences by consulting with a third reviewer. The following characteristics of eligible articles were extracted into the data extraction table: first author, year of publication, research design, sample, intervention characteristics (dose, duration of treatment, and so on), control, and outcome data. If the studies that included in this meta were not provided the original data in the text or table, we will reference the figures in the study or try to contact the original researchers for further information.

\section{Quality assessment}

Using the Cochrane risk-of-bias tool (version 5.1.0) (18), each trial was evaluated in the following 7 areas related to the risk of bias: (I) random sequence generation, (II) concealment of treatment allocation, (III) blinding of participants and personnel, (IV) blinding of outcome evaluation, (V) incomplete outcome data, (VI) selective reporting, and (VII) other bias. For each criterion, the score was assigned according to high, low, or ambiguous bias risks. Any differences in assessment were resolved through discussion with a third reviewer.

\section{Statistical analysis}

All meta-analyses were conducted with RevMan software (version 5.4). All outcomes are described and were analyzed as mean \pm standard deviation (SD). The mean difference (MD) and $95 \%$ confidence interval (CI) were used to measure all outcomes. If raw data were not reported, we imputed unreported means \pm SD using established methods via other information provided in the publication (19). Heterogeneity of the study was tested via chi-square $\left(\chi^{2}\right)$ based Cochran $\mathrm{Q}$ statistic and $\mathrm{I}^{2}$ statistic. In the case of heterogeneity, the random effects model was used; otherwise, a fixed effects model was used. Sensitivity analysis was performed to evaluate the reliability and stability of the combined results by deleting 1 trial at a time and repeating the meta-analysis. A funnel plot was used to judge publication bias (20).

\section{Results}

\section{Search results and study characteristics}

As shown in Figure 1, 314 studies were initially identified, and after screening of their titles and abstracts, 42 studies were selected for full-text review. Among those, 32 were excluded due to not being RCTs; having no quantitative outcomes; or being conference abstracts, reviews, or animal experiments. Finally, 10 studies (21-30) were enrolled in our meta-analysis.

As shown in Table 1, a total of 610 patients were identified according to the search strategy, among which 322 were divided into the $\mathrm{n}-3$ PUFAs group and the rest into the control group. The dosage of n-3 PUFA was 1000$3500 \mathrm{mg} / \mathrm{D}$ for $6-12$ weeks. The sample size ranged from 51 to 78 .

\section{Quality assessment}

The results of quality assessment of RCTs in our metaanalysis are shown in Figure 2. In 5 of the trials, the 


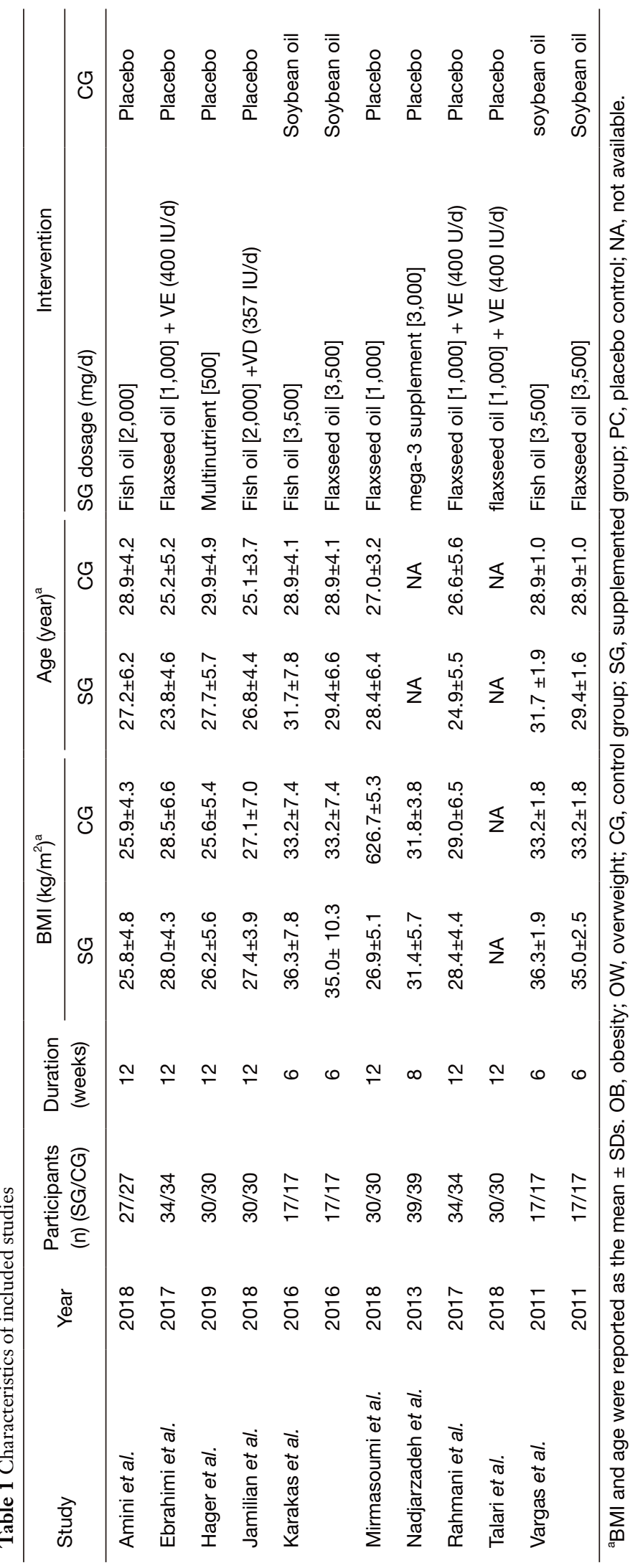


A

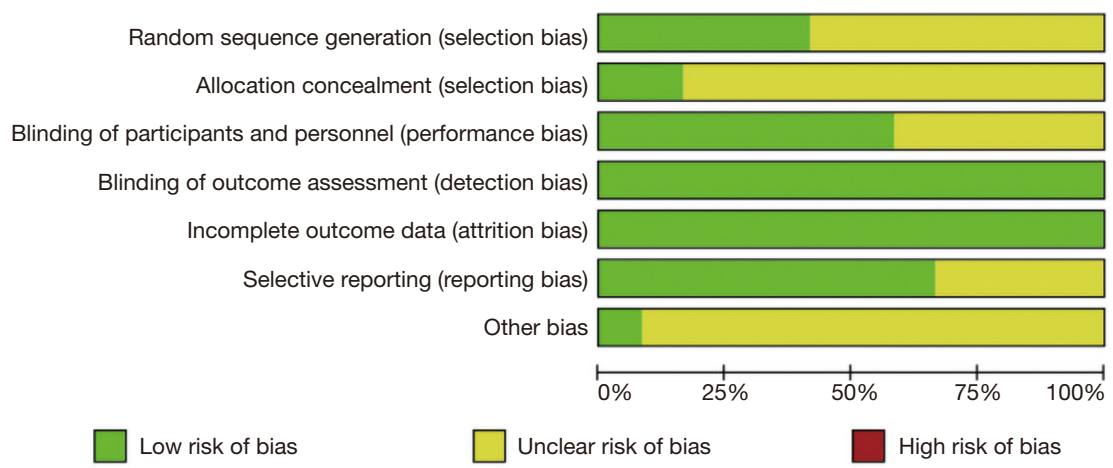

B

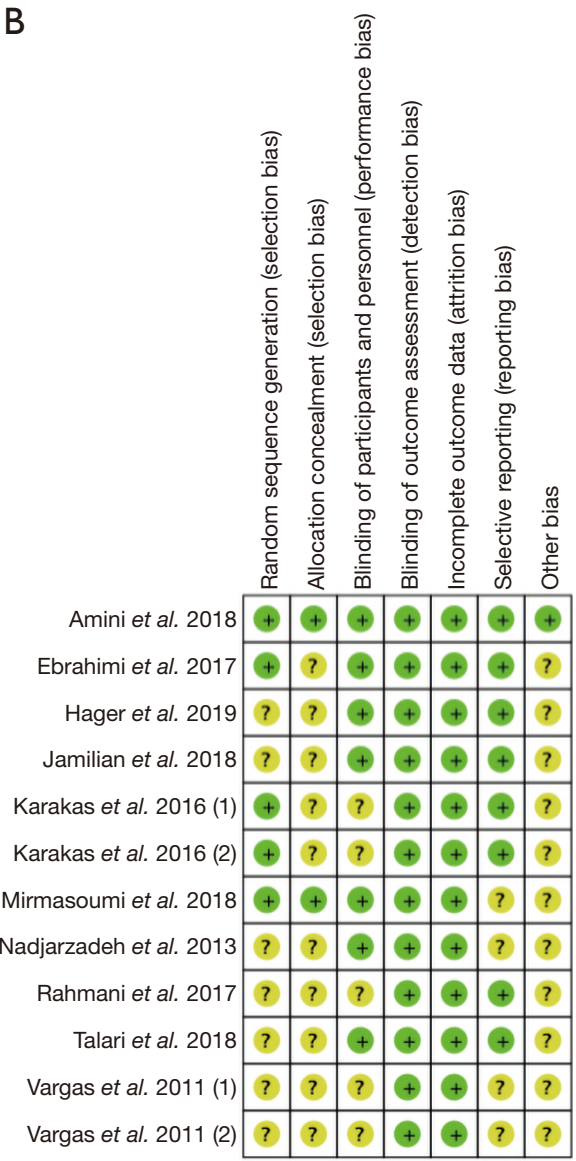

Figure 2 Researches quality (A) bias risk summary (B) overall assessment of bias risk.

patients were randomly divided into 2 groups according to a computer-generated random list or computer-generated random number pattern; the other 5 RCTs without randomization method details. In the allocation evaluation, 2 trials were classified as low-risk trials due to the appropriate use of allocation concealment; the allocation concealment was not specified in the remaining 8 trials. In our study, 7 RCTs were low risk and outcome assessment of blinding participants and personnel, and 3 trials were classified as ambiguous. In terms of the blind method for incomplete outcome data, all RCTs were low risk. In the evaluation of selective reporting, 7 trials were classified as low risk, and the remaining 3 were classified as unclear or with other biases.

\section{Pooled analysis}

Effect of n-3 PUFAs on CRP, GSH, MDA, and TAC Meta-analysis of 3 RCTs showed that n-3 PUFAs significantly altered CRP in PCOS (Figure 3: $-8.97 \mathrm{mg} / \mathrm{dL}$; $95 \%$ CI: -17.66 to $-0.28 \mathrm{mg} / \mathrm{dL} ; \mathrm{P}=0.04 ; \mathrm{I}^{2}=99 \%$ ).

The meta-analysis of 3 RCTs showed that n-3 PUFAs significantly altered GSH in PCOS (Figure 4: $-12.63 \mathrm{mg} / \mathrm{dL}$; $95 \%$ CI: -50.34 to $25.07 \mathrm{mg} / \mathrm{dL} ; \mathrm{P}=0.51 ; \mathrm{I}^{2}=56 \%$ ). Meta-analysis of 3 studies demonstrated that n-3 PUFAs significantly reduced serum MDA (Figure $5:-0.40 \mathrm{mg} / \mathrm{dL}$; $95 \%$ CI: -0.56 to $\left.-0.25 \mathrm{mg} / \mathrm{dL} ; \mathrm{P}<0.00001 ; \mathrm{I}^{2}=42 \%\right)$. The meta-analysis of 7 RCTs including 506 participants showed that n-3 PUFAs significantly changed TAC in PCOS (Figure 6: $72.24 \mathrm{mg} / \mathrm{dL}$; $95 \%$ CI: 22.32 to $122.16 \mathrm{mg} / \mathrm{dL}$; $\left.\mathrm{P}=0.005 ; \mathrm{I}^{2}=50 \%\right)$.

\section{Effect of n-3 PUFAs on DHEAS, FAI, FSH, LH, SHBG, and TT}

Results showed that n-3 PUFAs significantly reduced DHEAS in PCOS (Figure 7: $-0.01 \mathrm{mg} / \mathrm{dL} ; 95 \%$ CI: -1.53 to $\left.1.50 \mathrm{mg} / \mathrm{dL} ; \mathrm{P}=0.99 ; \mathrm{I}^{2}=78 \%\right)$. Meta-analysis showed 


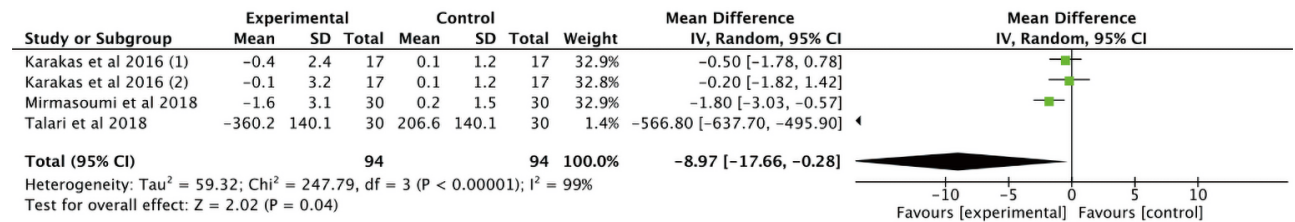

Figure 3 Effect of n-3 PUFAs on CRP. n-3 PUFAs, omega-3 polyunsaturated fatty acids; CRP, C-reactive protein; SD, standard deviation; CI, confidence interval.

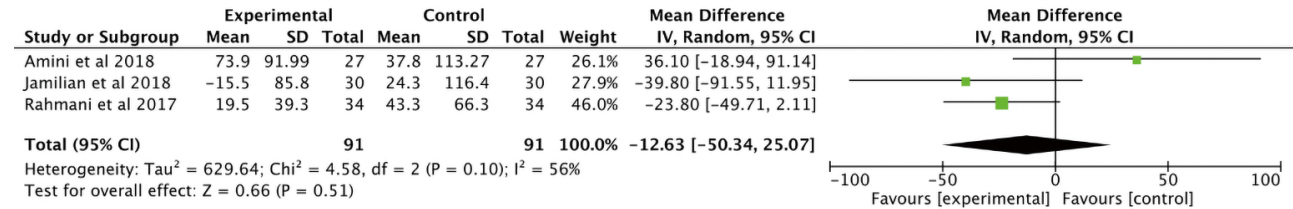

Figure 4 Effect of n-3 PUFAs on GSH. n-3 PUFAs, omega-3 polyunsaturated fatty acids; GSH, glutathione; SD, standard deviation; CI, confidence interval.

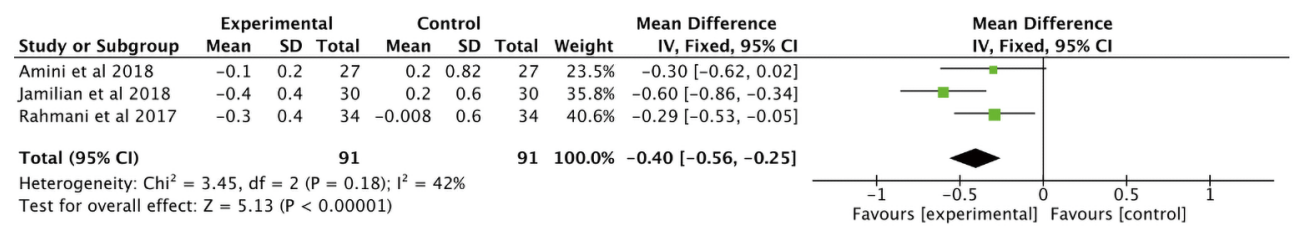

Figure 5 Effect of n-3 PUFAs on MDA. n-3 PUFAs, omega-3 polyunsaturated fatty acids; MDA, malondialdehyde; SD, standard deviation; CI, confidence interval.

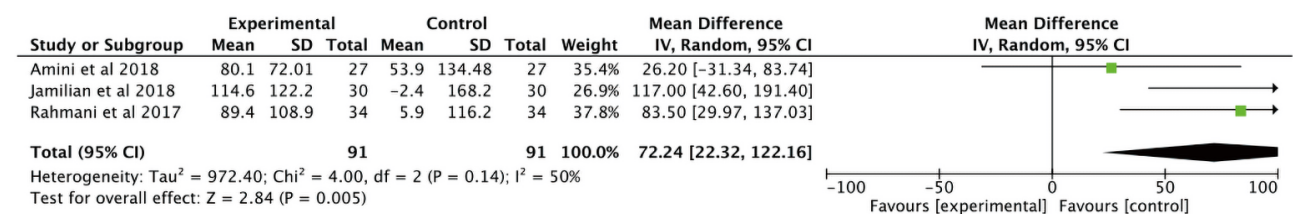

Figure 6 Effect of n-3 PUFAs on TAC. n-3 PUFAs, omega-3 polyunsaturated fatty acids; TAC, total antioxidant capacity; SD, standard deviation; CI, confidence interval.

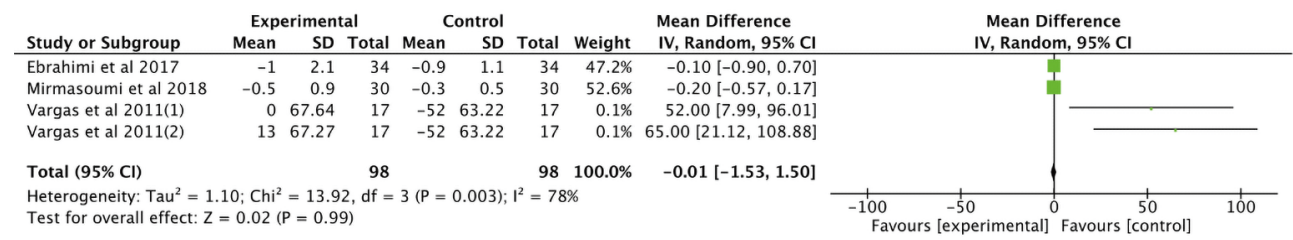

Figure 7 Effect of n-3 PUFAs on DHEAS. n-3 PUFAs, omega-3 polyunsaturated fatty acids; DHEAS, dehydroepiandrosterone sulfate; SD, standard deviation; CI, confidence interval. 


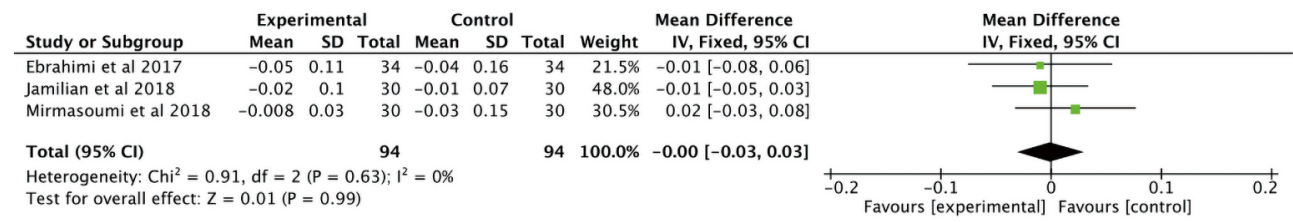

Figure 8 Effect of n-3 PUFAs on FAI. n-3 PUFAs, omega-3 polyunsaturated fatty acids; FAI, free androgen index; SD, standard deviation; CI, confidence interval.

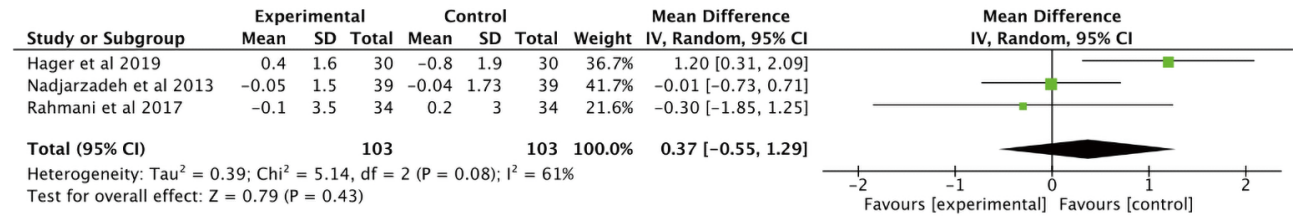

Figure 9 Effect of n-3 PUFAs on FSH. n-3 PUFAs, omega-3 polyunsaturated fatty acids; FSH, follicle stimulating hormone; SD, standard deviation; CI, confidence interval.

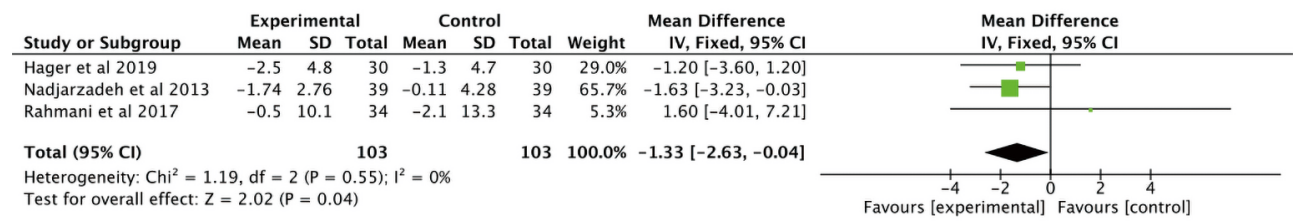

Figure 10 Effect of n-3 PUFAs on LH. n-3 PUFAs, omega-3 polyunsaturated fatty acids; LH, luteinizing hormone; SD, standard deviation; CI, confidence interval.

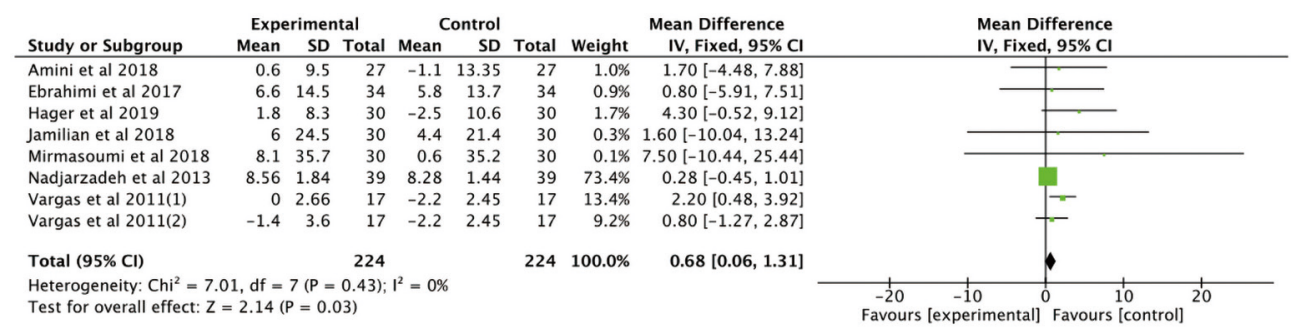

Figure 11 Effect of n-3 PUFAs on SHBG. n-3 PUFAs, omega-3 polyunsaturated fatty acids; SHBG, sex hormone binding globulin; SD, standard deviation; CI, confidence interval.

that n-3 PUFAs significantly changed serum FAI in PCOS patients (Figure 8: $0.00 \mathrm{mg} / \mathrm{dL} ; 95 \% \mathrm{CI}:-0.03$ to $\left.0.03 \mathrm{mg} / \mathrm{dL} ; \mathrm{P}=0.99 ; \mathrm{I}^{2}=0 \%\right)$. Meta-analysis demonstrated that n-3 PUFAs significantly reduced FSH (Figure 9: $0.37 \mathrm{mg} / \mathrm{dL} ; 95 \% \mathrm{CI}:-0.55$ to $\left.1.29 \mathrm{mg} / \mathrm{dL} ; \mathrm{P}=0.43 ; \mathrm{I}^{2}=61\right)$. This meta-analysis showed that n-3 PUFAs significantly changed serum LH in PCOS (Figure 10: $-1.33 \mathrm{mg} / \mathrm{dL}$;
95\% CI: -2.63 to $\left.-0.04 \mathrm{mg} / \mathrm{dL} ; \mathrm{P}=0.04 ; \mathrm{I}^{2}=0 \%\right)$. The metaanalysis of 8 RCTs with 448 participants showed that n-3 PUFAs had a significant effect on serum SHBG (Figure 11: $0.68 \mathrm{mg} / \mathrm{dL} ; 95 \%$ CI: 0.06 to $\left.1.31 \mathrm{mg} / \mathrm{dL} ; \mathrm{P}=0.03 ; \mathrm{I}^{2}=0 \%\right)$. Results demonstrated that n-3 PUFAs significantly improved serum TT in PCOS (Figure 12: $-0.11 \mathrm{mg} / \mathrm{dL}$; $95 \%$ CI: -0.18 to $\left.-0.04 \mathrm{mg} / \mathrm{dL} ; \mathrm{P}=0.02 ; \mathrm{I}^{2}=73 \%\right)$. 


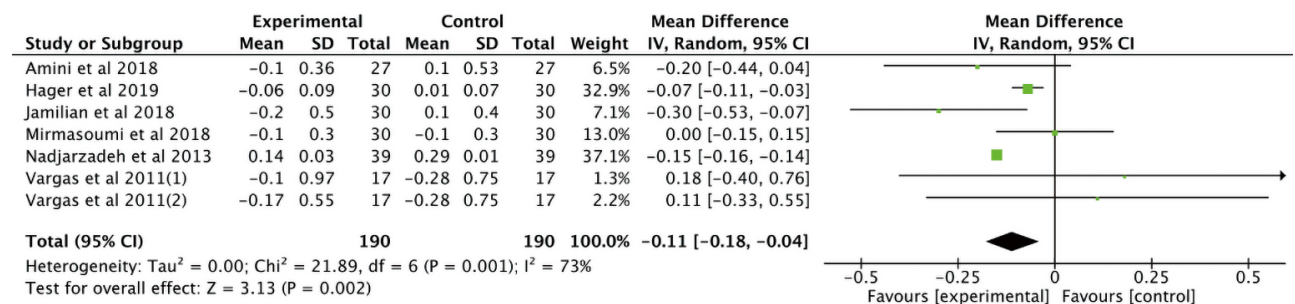

Figure 12 Effect of n-3 PUFAs on TT. n-3 PUFAs, omega-3 polyunsaturated fatty acids; TT, total testosterone; SD, standard deviation; CI, confidence interval.
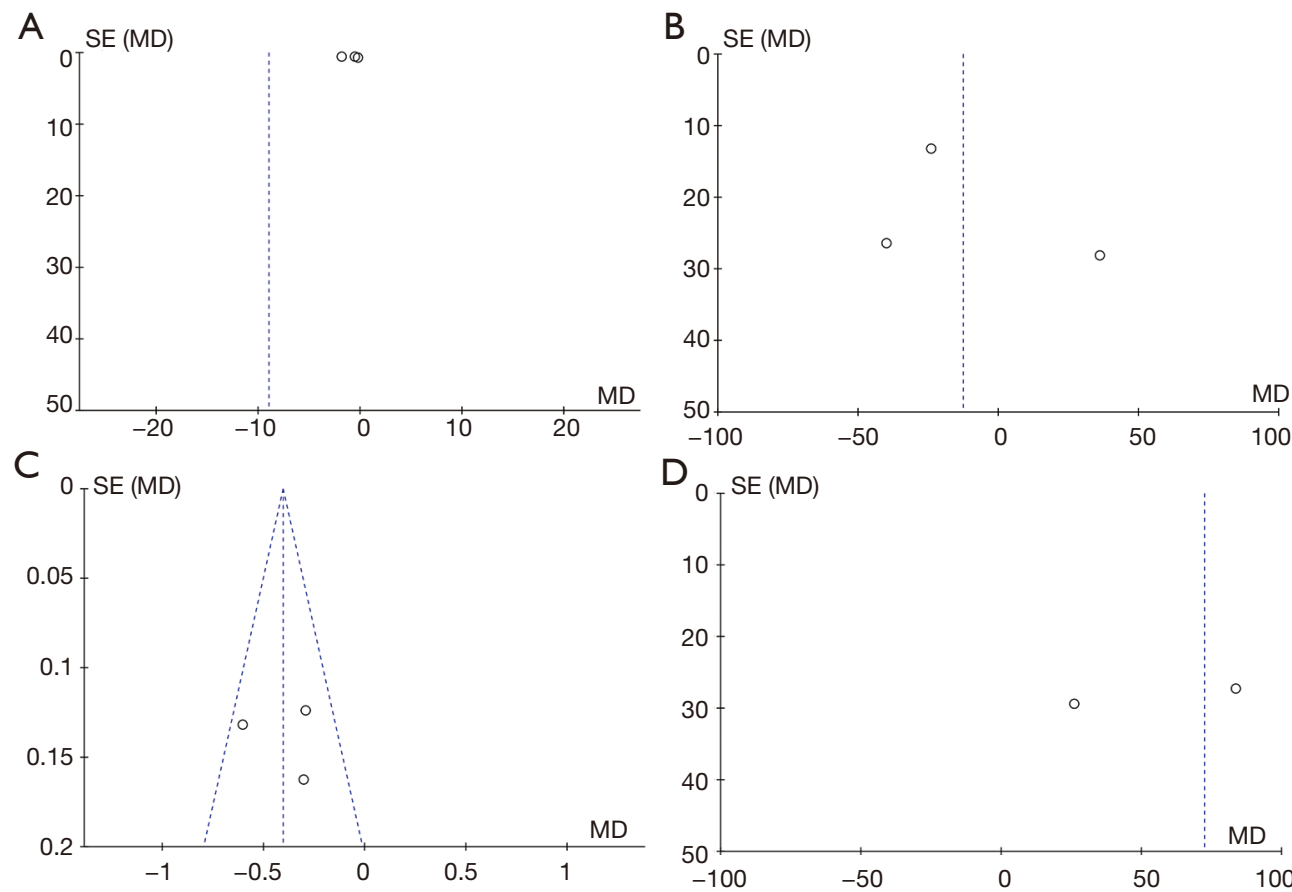

Figure 13 Funnel plot (A) CRP, (B) GSH, (C) MDA, and (D) TAC, detailing publication bias in the studies selected for analyses. CRP, C-reactive protein; GSH, glutathione; MDA, malondialdehyde; TAC, total antioxidant capacity.

\section{Publication bias}

According to the funnel plots, there was no obvious publication bias regarding CRP, GSH, MDA, TAC, DHEAS, FAI, FSH, LH, SHBG, or TT (Figures 13,14).

\section{Discussion}

The common endocrine disease PCOS is emerging as a major health problem. Recent studies have found that $n-3$ PUFAs can improve the hormone disorder of PCOS, but the results have been controversial. Thus, we systematically reviewed 10 available RCTs comprising 610 participants to evaluate the efficacy of n-3 PUFA therapy on hormones, oxidative stress, and inflammatory markers in women with PCOS. The current research demonstrated that n-3 PUFA treatment had significant reduced CRP, MDA, LH, TT, TAC, SHBG, but did not affect GSH, DHEAS, FAI, or FSH concentrations.

\section{Effect of n-3 PUFA supplementation on bormones}

PCOS has clinical heterogeneity, which is based on the influence of genetic and environmental factors (31). Hypothalamic amenorrhea (HA) is a key pathophysiological 

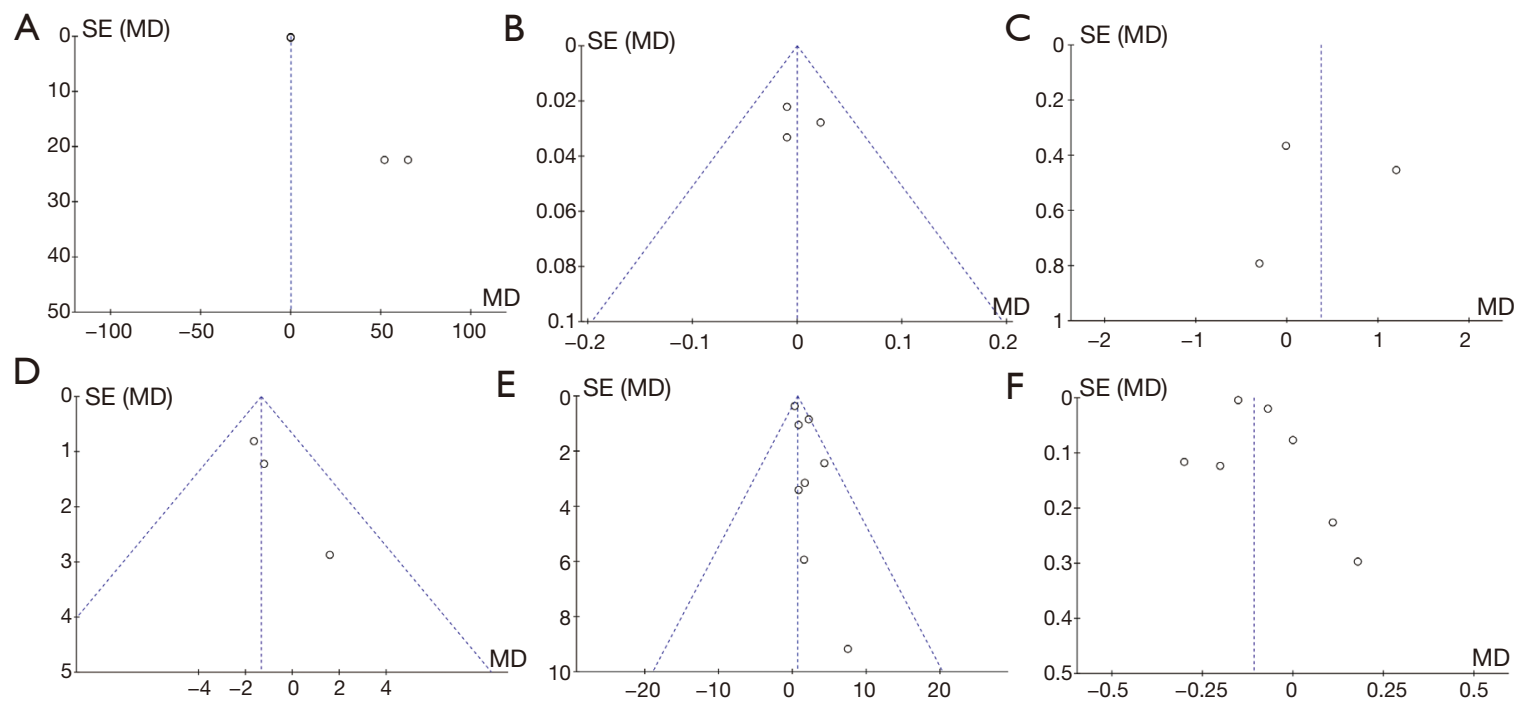

Figure 14 Funnel plot of SE by standardized mean difference for (A) DHEAS, (B) FAI, (C) FSH, (D) LH, (E) SHBG, and (F) TT, detailing publication bias in the studies selected for analyses. Closed circles represent observed published studies. n-3 PUFAs, omega-3 polyunsaturated fatty acids; DHEAS, dehydroepiandrosterone sulfate; FAI, free androgen index; FSH, follicle stimulating hormone; LH, luteinizing hormone; SHBG, sex hormone binding globulin; TT, total testosterone.

factor in PCOS patients. Ovarian dysfunction, abnormal ovulation, and irregular menstruation can be induced via high circulating androgen levels in local tissues and ovaries (2). In addition, some of the characteristics of PCOS involve hyperandrogen-like changes, such as hirsutism, acne, and seborrhea. Our results suggest that n-3 PUFA supplementation improves LH, TT, and SHBG levels in women with PCOS.

\section{Effects of n-3 PUFA supplementation on inflammation and oxidative stress}

Chronic inflammatory response and inflammatory mediators play a key part in the occurrence and development of IR, which directly affect the phosphorylation and activation of IRS- 1 and IRS-2, and cause IR (32). Nuclear transcription factor kappa-B (NF-KB) is a key transcription factor regulating inflammatory response (33). It has been demonstrated that n-3 PUFAs may lead to insulin sensitivity. Gray et al. researches have indicated that n-3 PUFA supplementation exerted good effects in PCOS patients (33). Our results suggested that n-3 PUFA supplementation improves CRP, MDA, and TAC levels in women with PCOS.

There were also some limitations to our research. First, few eligible studies were included in this study. Second, due to factors, such as there being different research groups, there was a high degree of heterogeneity, which might have led to a high risk of bias. Finally, the PCOS patients have different statuses, and the history of use of hypolipidemic drugs, such as statins, was unclear.

\section{Conclusions}

To date, our study has indicated that n-3 PUFA therapy has significant reduction effects on CRP, MDA, LH, TT, TAC, and SHBG, but does not affect GSH, DHEAS, FAI, or FSH concentrations. In considering the recommendation of n-3 PUFA supplementation, n-3 PUFAs may be a simple and low-risk add-on therapy for patients with PCOS. However, given the limitations in this study, the effects of n-3 PUFA on hormones, oxidative stress, and inflammation in PCOS patients should be further investigated.

\section{Acknowledgments}

Funding: None.

\section{Footnote}

Reporting Checklist: The authors have completed the PRISMA reporting checklist. Available at https://dx.doi. 
org/10.21037/apm-21-2018

Conflicts of Interest: All authors have completed the ICMJE uniform disclosure form (available at http://dx.doi.org/ apm-21-2018). The authors have no conflicts of interest to declare.

Ethical Statement: The authors are accountable for all aspects of the work in ensuring that questions related to the accuracy or integrity of any part of the work are appropriately investigated and resolved.

Open Access Statement: This is an Open Access article distributed in accordance with the Creative Commons AttributionNonCommercial-NoDerivs 4.0 International License (CC BYNC-ND 4.0), which permits the non-commercial replication and distribution of the article with the strict proviso that no changes or edits are made and the original work is properly cited (including links to both the formal publication through the relevant DOI and the license). See: https://creativecommons. org/licenses/by-nc-nd/4.0/.

\section{References}

1. Wekker V, van Dammen L, Koning A, et al. Long-term cardiometabolic disease risk in women with PCOS: a systematic review and meta-analysis. Hum Reprod Update 2020;26:942-60.

2. Goodarzi MO, Dumesic DA, Chazenbalk G, et al. Polycystic ovary syndrome: etiology, pathogenesis and diagnosis. Nat Rev Endocrinol 2011;7:219-31.

3. DeUgarte CM, Bartolucci AA, Azziz R. Prevalence of insulin resistance in the polycystic ovary syndrome using the homeostasis model assessment. Fertil Steril 2005;83:1454-60.

4. Ruan X, Li M, Mueck AO. Why does Polycystic Ovary Syndrome (PCOS) Need Long-term Management? Curr Pharm Des 2018;24:4685-92.

5. Anagnostis P, Tarlatzis BC, Kauffman RP. Polycystic ovarian syndrome (PCOS): Long-term metabolic consequences. Metabolism 2018;86:33-43.

6. Artimani T, Karimi J, Mehdizadeh M, et al. Evaluation of pro-oxidant-antioxidant balance (PAB) and its association with inflammatory cytokines in polycystic ovary syndrome (PCOS). Gynecol Endocrinol 2018;34:148-52.

7. Melo AS, Kliemchen J, Junior AA, et al. Oxidative stress and polycystic ovary syndrome: evaluation during ovarian stimulation for ICSI. Reproduction 2016. [Epub ahead of print]. doi: 10.1530/REP-16-0084.

8. Teede H, Deeks A, Moran L. Polycystic ovary syndrome: a complex condition with psychological, reproductive and metabolic manifestations that impacts on health across the lifespan. BMC Med 2010;8:41.

9. Blay SL, Aguiar JV, Passos IC. Polycystic ovary syndrome and mental disorders: a systematic review and exploratory meta-analysis. Neuropsychiatr Dis Treat 2016;12:2895-903.

10. Barrea L, Marzullo P, Muscogiuri G, et al. Source and amount of carbohydrate in the diet and inflammation in women with polycystic ovary syndrome. Nutr Res Rev 2018;31:291-301.

11. Sóter MO, Ferreira CN, Sales MF, et al. Peripheral bloodderived cytokine gene polymorphisms and metabolic profile in women with polycystic ovary syndrome. Cytokine 2015;76:227-35.

12. Osibogun O, Ogunmoroti O, Michos ED. Polycystic ovary syndrome and cardiometabolic risk: Opportunities for cardiovascular disease prevention. Trends Cardiovasc Med 2020;30:399-404.

13. Phelan N, O'Connor A, Kyaw Tun T, et al. Hormonal and metabolic effects of polyunsaturated fatty acids in young women with polycystic ovary syndrome: results from a cross-sectional analysis and a randomized, placebocontrolled, crossover trial. Am J Clin Nutr 2011;93:652-62.

14. Santos HO, Price JC, Bueno AA. Beyond Fish Oil Supplementation: The Effects of Alternative Plant Sources of Omega-3 Polyunsaturated Fatty Acids upon Lipid Indexes and Cardiometabolic Biomarkers-An Overview. Nutrients 2020;12:3159.

15. Oner G, Muderris II. Efficacy of omega-3 in the treatment of polycystic ovary syndrome. J Obstet Gynaecol 2013;33:289-91.

16. Kabir M, Skurnik G, Naour N, et al. Treatment for 2 mo with $\mathrm{n} 3$ polyunsaturated fatty acids reduces adiposity and some atherogenic factors but does not improve insulin sensitivity in women with type 2 diabetes: a randomized controlled study. Am J Clin Nutr 2007;86:1670-9.

17. Shamseer L, Moher D, Clarke M, et al. Preferred reporting items for systematic review and meta-analysis protocols (PRISMA-P) 2015: elaboration and explanation. BMJ 2015;350:g7647.

18. Jørgensen L, Paludan-Müller AS, Laursen DR, et al. Evaluation of the Cochrane tool for assessing risk of bias in randomized clinical trials: overview of published comments and analysis of user practice in Cochrane and nonCochrane reviews. Syst Rev 2016;5:80.

19. Wan X, Wang W, Liu J, et al. Estimating the sample mean 
and standard deviation from the sample size, median, range and/or interquartile range. BMC Med Res Methodol 2014;14:135.

20. Duval S, Tweedie R. Trim and fill: A simple funnel-plotbased method of testing and adjusting for publication bias in meta-analysis. Biometrics 2000;56:455-63.

21. Amini M, Bahmani F, Foroozanfard F, et al. The effects of fish oil omega-3 fatty acid supplementation on mental health parameters and metabolic status of patients with polycystic ovary syndrome: a randomized, double-blind, placebo-controlled trial. J Psychosom Obstet Gynaecol 2018. [Epub ahead of print]. doi: 10.1080/0167482X.2018.1508282.

22. Ebrahimi FA, Samimi M, Foroozanfard F, et al. The Effects of Omega-3 Fatty Acids and Vitamin E CoSupplementation on Indices of Insulin Resistance and Hormonal Parameters in Patients with Polycystic Ovary Syndrome: A Randomized, Double-Blind, PlaceboControlled Trial. Exp Clin Endocrinol Diabetes 2017;125:353-9.

23. Hager M, Nouri K, Imhof $M$, et al. The impact of a standardized micronutrient supplementation on PCOStypical parameters: a randomized controlled trial. Arch Gynecol Obstet 2019;300:455-60.

24. Jamilian M, Samimi M, Mirhosseini N, et al. The influences of vitamin D and omega-3 co-supplementation on clinical, metabolic and genetic parameters in women with polycystic ovary syndrome. J Affect Disord 2018;238:32-8.

25. Karakas SE, Perroud B, Kind T, et al. Changes in plasma metabolites and glucose homeostasis during omega-3 polyunsaturated fatty acid supplementation in women with polycystic ovary syndrome. BBA Clin 2016;5:179-85.

26. Mirmasoumi G, Fazilati M, Foroozanfard F, et al. The Effects of Flaxseed Oil Omega-3 Fatty Acids Supplementation on Metabolic Status of Patients with Polycystic Ovary Syndrome: A Randomized, Double-
Blind, Placebo-Controlled Trial. Exp Clin Endocrinol Diabetes 2018;126:222-8.

27. Nadjarzadeh A, Dehghani Firouzabadi R, Vaziri N, et al. The effect of omega-3 supplementation on androgen profile and menstrual status in women with polycystic ovary syndrome: A randomized clinical trial. Iran J Reprod Med 2013;11:665-72.

28. Rahmani E, Samimi M, Ebrahimi FA, et al. The effects of omega-3 fatty acids and vitamin E co-supplementation on gene expression of lipoprotein(a) and oxidized low-density lipoprotein, lipid profiles and biomarkers of oxidative stress in patients with polycystic ovary syndrome. Mol Cell Endocrinol 2017;439:247-55.

29. Talari HR, Poladchang S, Hamidian Y, et al. The Effects of Omega-3 and Vitamin E Co-supplementation on Carotid Intima-media Thickness and Inflammatory Factors in Patients with Polycystic Ovary Syndrome. Oman Med J 2018;33:473-9.

30. Vargas ML, Almario RU, Buchan W, et al. Metabolic and endocrine effects of long-chain versus essential omega-3 polyunsaturated fatty acids in polycystic ovary syndrome. Metabolism 2011;60:1711-8.

31. Lorenz LB, Wild RA. Polycystic ovarian syndrome: an evidence-based approach to evaluation and management of diabetes and cardiovascular risks for today's clinician. Clin Obstet Gynecol 2007;50:226-43.

32. Zuo T, Zhu M, Xu W, et al. Iridoids with Genipin Stem Nucleus Inhibit Lipopolysaccharide-Induced Inflammation and Oxidative Stress by Blocking the NF- B Pathway in Polycystic Ovary Syndrome. Cell Physiol Biochem 2017;43:1855-65.

33. Gray B, Steyn F, Davies PS, et al. Omega-3 fatty acids: a review of the effects on adiponectin and leptin and potential implications for obesity management. Eur J Clin Nutr 2013;67:1234-42.

(English Language Editor: J. Jones)
Cite this article as: Yuan J, Wen X, Jia M. Efficacy of omega-3 polyunsaturated fatty acids on hormones, oxidative stress, and inflammatory parameters among polycystic ovary syndrome: a systematic review and meta-analysis. Ann Palliat Med 2021;10(8):8991-9001. doi: 10.21037/apm-21-2018 